\title{
Search for Active-Sterile Antineutrino Mixing Using Neutral-Current Interactions with the NOvA Experiment
}

M. A. Acero, ${ }^{2}$ P. Adamson, ${ }^{12}$ L. Aliaga, ${ }^{12}$ N. Anfimov, ${ }^{26}$ A. Antoshkin, ${ }^{26}$ E. Arrieta-Diaz, ${ }^{28}$ L. Asquith, ${ }^{41}$ A. Aurisano, ${ }^{6}$ A. Back, ${ }^{20,24}$ C. Backhouse, ${ }^{45}$ M. Baird, ${ }^{20,41,46}$ N. Balashov, ${ }^{26}$ P. Baldi, ${ }^{25}$ B. A. Bambah, ${ }^{17}$ S. Bashar, ${ }^{44}$ K. Bays, ${ }^{4,19}$ R. Bernstein, ${ }^{12}$ V. Bhatnagar, ${ }^{34}$ B. Bhuyan, ${ }^{14}$ J. Bian, ${ }^{25,31}$ J. Blair, ${ }^{16}$ A. C. Booth, ${ }^{41}$ R. Bowles,${ }^{20}$ C. Bromberg, ${ }^{29}$ N. Buchanan, ${ }^{8}$ A. Butkevich, ${ }^{22}$ S. Calvez, ${ }^{8}$ T. J. Carroll1, ${ }^{43,49}$ E. Catano-Mur, ${ }^{48}$ B. C. Choudhary, ${ }^{10}$ A. Christensen, ${ }^{8}$ T. E. Coan, ${ }^{39}$ M. Colo, ${ }^{48}$ L. Cremonesi,${ }^{36,45}$ G. S. Davies, ${ }^{32,20}$ P. F. Derwent, ${ }^{12}$ P. Ding, ${ }^{12}$ Z. Djurcic, ${ }^{1}$ M. Dolce, ${ }^{44}$ D. Doyle, ${ }^{8}$ D. Dueñas Tonguino, ${ }^{6}$ E. C. Dukes,${ }^{46}$ H. Duyang, ${ }^{38}$ S. Edayath, ${ }^{7}$ R. Ehrlich,${ }^{46}$ M. Elkins,${ }^{24}$ E. Ewart, ${ }^{20}$ G. J. Feldman, ${ }^{15}$ P. Filip, ${ }^{23}$ J. Franc, ${ }^{9}$ M. J. Frank, ${ }^{37}$ H. R. Gallagher, ${ }^{44}$ R. Gandrajula, ${ }^{29,46}$ F. Gao, ${ }^{35}$ A. Giri, ${ }^{18}$ R. A. Gomes, ${ }^{13}$ M. C. Goodman, ${ }^{1}$ V. Grichine, ${ }^{27}$ M. Groh,${ }^{8,20}$ R. Group ${ }^{46}$ B. Guo, ${ }^{38}$ A. Habig, ${ }^{30}$ F. Hakl, ${ }^{21}$ A. Hall, ${ }^{46}$ J. Hartnell, ${ }^{41}$ R. Hatcher, ${ }^{12}$ H. Hausner, ${ }^{49}$ K. Heller, ${ }^{31}$ J. Hewes, ${ }^{6}$ A. Himmel, ${ }^{12}$ A. Holin, ${ }^{45}$ J. Huang ${ }^{43}$ B. Jargowsky, ${ }^{25}$ J. Jarosz, ${ }^{8}$ F. Jediny, ${ }^{9}$ C. Johnson, ${ }^{8}$ M. Judah, ${ }^{8,35}$ I. Kakorin ${ }^{26}$ D. Kalra ${ }^{34}$ A. Kalitkina ${ }^{26}$ D. M. Kaplan, ${ }^{19}$ R. Keloth, ${ }^{7}$ O. Klimov, ${ }^{26}$ L. W. Koerner, ${ }^{16}$ L. Kolupaeva, ${ }^{26}$ S. Kotelnikov, ${ }^{27}$ R. Kralik, ${ }^{41}$ Ch. Kullenberg, ${ }^{26}$ M. Kubu, ${ }^{9}$ A. Kumar, ${ }^{34}$ C. D. Kuruppu, ${ }^{38}$ V. Kus, ${ }^{9}$ T. Lackey, ${ }^{20}$ P. Lasorak, ${ }^{41}$ K. Lang, ${ }^{43}$ J. Lesmeister, ${ }^{16}$ S. Lin, ${ }^{8}$ A. Lister, ${ }^{49}$ J. Liu, ${ }^{25}$ M. Lokajicek, ${ }^{23}$ S. Magill, ${ }^{1}$ M. Manrique Plata, ${ }^{20}$ W. A. Mann, ${ }^{44}$ M. L. Marshak, ${ }^{31}$ M. Martinez-Casales, ${ }^{24}$ V. Matveev, ${ }^{22}$ B. Mayes, ${ }^{41}$ D. P. Méndez,${ }^{41}$ M. D. Messier, ${ }^{20}$ H. Meyer, ${ }^{47}$ T. Miao, ${ }^{12}$ W. H. Miller, ${ }^{31}$ S. R. Mishra, ${ }^{38}$ A. Mislivec, ${ }^{31}$ R. Mohanta, ${ }^{17}$ A. Moren,${ }^{30}$ A. Morozova, ${ }^{26}$ W. Mu, ${ }^{12}$ L. Mualem, ${ }^{4}$ M. Muether, ${ }^{47}$ K. Mulder, ${ }^{45}$ D. Naples,${ }^{35}$ N. Nayak, ${ }^{25}$ J. K. Nelson, ${ }^{48}$ R. Nichol, ${ }^{45}$ E. Niner, ${ }^{12}$ A. Norman, ${ }^{12}$ A. Norrick, ${ }^{12}$ T. Nosek, ${ }^{5}$ H. Oh ${ }^{6}$ A. Olshevskiy, ${ }^{26}$ T. Olson, ${ }^{44}$ J. Ott, ${ }^{25}$ J. Paley, ${ }^{12}$ R. B. Patterson, ${ }^{4}$ G. Pawloski, ${ }^{31}$ O. Petrova, ${ }^{26}$ R. Petti ${ }^{38}$ D. D. Phan, ${ }^{43,45}$ R. K. Plunkett, ${ }^{12}$ J. C. C. Porter, ${ }^{41}$ A. Rafique, ${ }^{1}$ V. Raj, ${ }^{4}$ M. Rajaoalisoa, ${ }^{6}$ B. Ramson, ${ }^{12}$ B. Rebel,${ }^{12,49}$ P. Rojas, ${ }^{8}$ V. Ryabov, ${ }^{27}$ O. Samoylov, ${ }^{26}$ M. C. Sanchez, ${ }^{24}$ S. Sánchez Falero, ${ }^{24}$ P. Shanahan, ${ }^{12}$ A. Sheshukov, ${ }^{26}$ P. Singh, ${ }^{10}$ V. Singh, ${ }^{3}$ E. Smith, ${ }^{20}$ J. Smolik, ${ }^{9}$ P. Snopok, ${ }^{19}$ N. Solomey, ${ }^{47}$ A. Sousa, ${ }^{6}$ K. Soustruznik, ${ }^{5}$ M. Strait,${ }^{31}$ L. Suter, ${ }^{12}$ A. Sutton, ${ }^{46}$ S. Swain, ${ }^{33}$ C. Sweeney, ${ }^{45}$ B. Tapia Oregui, ${ }^{43}$ P. Tas, ${ }^{5}$ T. Thakore, ${ }^{6}$ R. B. Thayyullathil, ${ }^{7}$ J. Thomas, ${ }^{45,49}$ E. Tiras,${ }^{11,24}$ J. Tripathi,${ }^{34}$ J. Trokan-Tenorio, ${ }^{48}$ A. Tsaris,${ }^{12}$ Y. Torun,${ }^{19}$ J. Urheim, ${ }^{20}$ P. Vahle, ${ }^{48}$ Z. Vallari, ${ }^{4}$ J. Vasel,${ }^{20}$ P. Vokac, ${ }^{9}$ T. Vrba,${ }^{9}$ M. Wallbank $\odot,{ }^{6, *}$ T. K. Warburton, ${ }^{24}$ M. Wetstein, ${ }^{24}$

D. Whittington, ${ }^{42,20}$ D. A. Wickremasinghe, ${ }^{12}$ S. G. Wojcicki, ${ }^{40}$ J. Wolcott ${ }^{44}$ W. Wu, ${ }^{25}$ Y. Xiao, ${ }^{25}$ A. Yallappa Dombara, ${ }^{42}$ K. Yonehara, ${ }^{12}$ S. Yu, ${ }^{1,19}$ Y. Yu, ${ }^{19}$ S. Zadorozhnyy, ${ }^{22}$ J. Zalesak, ${ }^{23}$ Y. Zhang, ${ }^{41}$ and R. Zwaska ${ }^{12}$

\section{(The NOvA Collaboration)}

\author{
${ }^{1}$ Argonne National Laboratory, Argonne, Illinois 60439, USA \\ ${ }^{2}$ Universidad del Atlantico, Carrera 30 No. 8-49, Puerto Colombia, Atlantico, Colombia \\ ${ }^{3}$ Department of Physics, Institute of Science, Banaras Hindu University, Varanasi, 221 005, India \\ ${ }^{4}$ California Institute of Technology, Pasadena, California 91125, USA \\ ${ }^{5}$ Charles University, Faculty of Mathematics and Physics, Institute of Particle and Nuclear Physics, Prague, Czech Republic \\ ${ }^{6}$ Department of Physics, University of Cincinnati, Cincinnati, Ohio 45221, USA \\ ${ }^{7}$ Department of Physics, Cochin University of Science and Technology, Kochi 682 022, India \\ ${ }^{8}$ Department of Physics, Colorado State University, Fort Collins, Colorado 80523-1875, USA \\ ${ }^{9}$ Czech Technical University in Prague, Brehova 7, 11519 Prague 1, Czech Republic \\ ${ }^{10}$ Department of Physics and Astrophysics, University of Delhi, Delhi 110007, India \\ ${ }^{11}$ Department of Physics, Erciyes University, Kayseri 38030, Turkey \\ ${ }^{12}$ Fermi National Accelerator Laboratory, Batavia, Illinois 60510, USA \\ ${ }^{13}$ Instituto de Física, Universidade Federal de Goiás, Goiánia, Goiás, 74690-900, Brazil \\ ${ }^{14}$ Department of Physics, IIT Guwahati, Guwahati, 781 039, India \\ ${ }^{15}$ Department of Physics, Harvard University, Cambridge, Massachusetts 02138, USA \\ ${ }^{16}$ Department of Physics, University of Houston, Houston, Texas 77204, USA \\ ${ }^{17}$ School of Physics, University of Hyderabad, Hyderabad, 500 046, India \\ ${ }^{18}$ Department of Physics, IIT Hyderabad, Hyderabad, 502 205, India \\ ${ }^{19}$ Illinois Institute of Technology, Chicago Illinois 60616, USA \\ ${ }^{20}$ Indiana University, Bloomington, Indiana 47405, USA \\ ${ }^{21}$ Institute of Computer Science, The Czech Academy of Sciences, 18207 Prague, Czech Republic \\ ${ }^{22}$ Institute for Nuclear Research of Russia, Academy of Sciences 7a, 60th October Anniversary prospect, Moscow 117312, Russia \\ ${ }^{23}$ Institute of Physics, The Czech Academy of Sciences, 18221 Prague, Czech Republic
}




\author{
${ }^{24}$ Department of Physics and Astronomy, Iowa State University, Ames, Iowa 50011, USA \\ ${ }^{25}$ Department of Physics and Astronomy, University of California at Irvine, Irvine, California 92697, USA \\ ${ }^{26}$ Joint Institute for Nuclear Research, Dubna, Moscow region 141980, Russia \\ ${ }^{27}$ Nuclear Physics and Astrophysics Division, Lebedev Physical Institute, Leninsky Prospect 53, 119991 Moscow, Russia \\ ${ }^{28}$ Universidad del Magdalena, Carrera 32 No 22 - 08 Santa Marta, Colombia \\ ${ }^{29}$ Department of Physics and Astronomy, Michigan State University, East Lansing, Michigan 48824, USA \\ ${ }^{30}$ Department of Physics and Astronomy, University of Minnesota Duluth, Duluth, Minnesota 55812, USA \\ ${ }^{31}$ School of Physics and Astronomy, University of Minnesota Twin Cities, Minneapolis, Minnesota 55455, USA \\ ${ }^{32}$ University of Mississippi, University, Mississippi 38677, USA \\ ${ }^{33}$ National Institute of Science Education and Research, Khurda, 752050, Odisha, India \\ ${ }^{34}$ Department of Physics, Panjab University, Chandigarh, 160 014, India \\ ${ }^{35}$ Department of Physics, University of Pittsburgh, Pittsburgh, Pennsylvania 15260, USA \\ ${ }^{36}$ School of Physics and Astronomy, Queen Mary University of London, London E1 4NS, United Kingdom \\ ${ }^{37}$ Department of Physics, University of South Alabama, Mobile, Alabama 36688, USA \\ ${ }^{38}$ Department of Physics and Astronomy, University of South Carolina, Columbia, South Carolina 29208, USA \\ ${ }^{39}$ Department of Physics, Southern Methodist University, Dallas, Texas 75275, USA \\ ${ }^{40}$ Department of Physics, Stanford University, Stanford, California 94305, USA \\ ${ }^{41}$ Department of Physics and Astronomy, University of Sussex, Falmer, Brighton BN1 9QH, United Kingdom \\ ${ }^{42}$ Department of Physics, Syracuse University, Syracuse New York 13210, USA \\ ${ }^{43}$ Department of Physics, University of Texas at Austin, Austin, Texas 78712, USA \\ ${ }^{44}$ Department of Physics and Astronomy, Tufts University, Medford, Massachusetts 02155, USA \\ ${ }^{45}$ Physics and Astronomy Department, University College London, Gower Street, London WC1E 6BT, United Kingdom \\ ${ }^{46}$ Department of Physics, University of Virginia, Charlottesville, Virginia 22904, USA \\ ${ }^{47}$ Department of Mathematics, Statistics, and Physics, Wichita State University, Wichita, Kansas 67206, USA \\ ${ }^{48}$ Department of Physics, William and Mary, Williamsburg, Virginia 23187, USA \\ ${ }^{49}$ Department of Physics, University of Wisconsin-Madison, Madison, Wisconsin 53706, USA
}

(Received 10 June 2021; accepted 30 September 2021; published 11 November 2021)

This Letter reports results from the first long-baseline search for sterile antineutrinos mixing in an accelerator-based antineutrino-dominated beam. The rate of neutral-current interactions in the two NOvA detectors, at distances of 1 and $810 \mathrm{~km}$ from the beam source, is analyzed using an exposure of $12.51 \times$ $10^{20}$ protons-on-target from the NuMI beam at Fermilab running in antineutrino mode. A total of 121 of neutral-current candidates are observed at the far detector, compared to a prediction of $122 \pm 11$ (stat.) \pm 15(syst.) assuming mixing only between three active flavors. No evidence for $\bar{\nu}_{\mu} \rightarrow \bar{\nu}_{s}$ oscillation is observed. Interpreting this result within a $3+1$ model, constraints are placed on the mixing angles $\theta_{24}<$ $25^{\circ}$ and $\theta_{34}<32^{\circ}$ at the $90 \%$ C.L. for $0.05 \mathrm{eV}^{2} \leq \Delta m_{41}^{2} \leq 0.5 \mathrm{eV}^{2}$, the range of mass splittings that produces no significant oscillations at the near detector. These are the first $3+1$ confidence limits set using long-baseline accelerator antineutrinos.

DOI: 10.1103/PhysRevLett.127.201801

Studies of neutrinos and antineutrinos from a variety of sources, including accelerators, the atmosphere, the Sun, and nuclear reactors [1-11], provide substantial evidence for mixing between the three known active flavors $\stackrel{(-)}{\nu}_{e}, \stackrel{(-)}{\nu}$, and $\stackrel{(-)}{\nu}_{\tau}$. However, the observation of antineutrinos in short-baseline oscillations [12,13], reactor [14,15], and gallium-based radiochemical experiments $[16,17]$ found inconsistency with this three-flavor model. These anomalous

Published by the American Physical Society under the terms of the Creative Commons Attribution 4.0 International license. Further distribution of this work must maintain attribution to the author(s) and the published article's title, journal citation, and DOI. Funded by SCOAP ${ }^{3}$. results may be explained by extending the mixing model to include more massive neutrino and antineutrino states in addition to $\stackrel{(-)}{\nu}_{1}, \stackrel{(-)}{\nu}_{2}$, and $\stackrel{(-)}{\nu}_{3}$ with new mass splittings large enough to provide oscillations over much shorter baselines than is possible with the known three-flavor mass differences. However, observations of the width of the $Z^{0}$-boson resonance at the LEP experiments put the constraint on the number of light antineutrinos participating in the weak interaction to be three [18]. Any additional antineutrinos of mass less than approximately half the $Z^{0}$ mass must, therefore, be sterile [19].

The simplest extension to this three-flavor model is referred to as the $3+1$ model and introduces a single new mass state, $\stackrel{(-)}{\nu}_{4}$, with a corresponding sterile flavor state, 


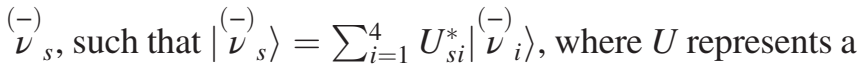
unitary $4 \times 4$ extended Pontecorvo-Maki-NagakawaSakata matrix [20,21]. The matrix can be parametrized as $U=R_{34} S_{24} S_{14} R_{23} S_{13} R_{12}$ [22], where $R_{i j}$ represents a rotation through mixing angle $\theta_{i j}$, and $S_{i j}$ represents a complex rotation through angle $\theta_{i j}$ and phase $\delta_{i j}$. In addition to the three-flavor mixing parameters and a new independent mass splitting $\Delta m_{41}^{2}=m_{4}^{2}-m_{1}^{2}$, this model includes three new mixing angles, $\theta_{14}, \theta_{24}$, and $\theta_{34}$, and two additional phases, $\delta_{14}$ and $\delta_{24}$, which may violate $C P$ symmetry. A number of atmospheric, long-baseline accelerator and reactor neutrino experiments have searched for oscillations outside of the three-flavor mixing framework and found no evidence of such processes [23-29], placing constraints on the allowed values of the parameters governing $3+1$ oscillations. Equivalent constraints have not yet been published from similar investigations of antineutrinos, the focus of the analysis described in this Letter. This is pertinent as the discrepancies observed in LSND [12] and MiniBooNE [30] involved measurements of oscillations from antineutrino beams, with different behavior reported in subsequent analyses of oscillations of neutrinos [31]. Further investigation is required to understand the oscillations of antineutrinos into potential sterile states.

NOvA can search for evidence of active to sterile oscillations through an analysis of the rate of neutralcurrent (NC) interactions in its near detector (ND) and far detector (FD) [29]. When oscillations occur between only the three active flavors, the rate of $\mathrm{NC}$ interactions is independent of flavor and is, thus, unaffected by oscillations. If the antineutrinos transition into an unseen sterile flavor state during propagation, a reduction in the number of $\mathrm{NC}$ interactions would be observed with a probability given approximately by [32]

$$
\begin{aligned}
1-P\left(\stackrel{(-)}{\nu}_{\mu} \rightarrow \stackrel{(-)}{\nu}_{s}\right) \approx & 1-c_{14}^{4} c_{34}^{2} \sin ^{2} 2 \theta_{24} \sin ^{2} \Delta_{41} \\
& -A \sin ^{2} \Delta_{31} \pm B \sin 2 \Delta_{31},
\end{aligned}
$$

where $c_{i j}=\cos \theta_{i j}, \quad \Delta_{j i}=\left(\Delta m_{j i}^{2} L / 4 E\right), \quad$ and $\quad \Delta m_{j i}^{2}=$ $m_{j}^{2}-m_{i}^{2}$. To first order, $A=\sin ^{2} \theta_{34} \sin ^{2} 2 \theta_{23}$ and $B=\frac{1}{2} \sin \delta_{24} \sin \theta_{24} \sin 2 \theta_{34} \sin 2 \theta_{23}$. This approximation assumes $\Delta_{21}=0, \sin ^{2} 2 \theta_{23}$ is near maximal, and considers the fast oscillation regime with limited detector resolution such that $\sin ^{2} \Delta_{41}=\frac{1}{2}$; an exact formalism, including matter effects, is used in the analysis. Studying the disappearance of $\mathrm{NC}$ events over a long baseline is, therefore, sensitive to the $3+1$ parameters $\theta_{24}, \theta_{34}, \delta_{24}$, and $\Delta m_{41}^{2}$.

Differences between neutrino and antineutrino oscillations due to $C P$ violation in this approximation are described by the $\delta_{24}$ phase and, therefore, the sign of the $B$ term, and are at most around $10 \%$ in the peak antineutrino flux region (around $2 \mathrm{GeV}$ ), with a maximum of
$20 \%$ at higher energies. While an additional asymmetry results from neutrinos gaining an extra effective mass due to forward scattering in matter, this effect is significantly smaller than that from the phase.

The $\Delta m_{41}^{2}$ parameter determines the frequency of the oscillations. For sufficiently small values, the oscillations develop over longer baselines than the ND and are more rapid than can be resolved at the FD, resulting in an average reduction in the $\mathrm{NC}$ rate over the energy spectrum. As the mass splitting increases, oscillations develop over shorter baselines and are evident at the ND. This analysis considers oscillations driven by $0.05 \mathrm{eV}^{2} \leq \Delta m_{41}^{2} \leq 0.5 \mathrm{eV}^{2}$, where the NC rate is unaffected at the ND and reduced at the FD.

The NOvA experiment consists of two functionally identical detectors placed in the NuMI beam [33]. The ND is located at Fermilab in Batavia, Illinois, $1 \mathrm{~km}$ from the NuMI target and $100 \mathrm{~m}$ underground; the FD is located near Ash River, Minnesota, $810 \mathrm{~km}$ from the beam source with a $3 \mathrm{~m}$ water-equivalent overburden of concrete and barite. The detectors are placed $14.6 \mathrm{mrad}$ off the axis of the beam to produce a narrow neutrino or antineutrino energy distribution peaked at $2.0 \mathrm{GeV}$ at the FD with a width of $0.4 \mathrm{GeV}$ and a high energy tail of around $10 \%$ of the peak.

The NuMI beam is produced from $120 \mathrm{GeV}$ protons incident on a mostly carbon target. Two magnetic focusing horns pulsed at $200 \mathrm{kA}$ focus the secondary pions and kaons, which subsequently decay in flight over $705 \mathrm{~m}$, including a $675 \mathrm{~m}$ decay pipe, to mainly muons and muon (anti)neutrinos. By selecting the current polarity in the horns, the beam can be run in a mode dominated by either $\nu_{\mu}$ or $\bar{\nu}_{\mu}$. The beam is extracted for $10 \mu$ s approximately every 1.3 seconds. A previous analysis searched for evidence of $\mathrm{NC}$ disappearance in a $\nu$-dominated beam [29]; this Letter is the first using the NOvA $\bar{\nu}$-dominated dataset. Across the $0-20 \mathrm{GeV}$ energy range, beam interactions at the ND are predicted to consist of $63 \% \bar{\nu}_{\mu}, 35 \% \nu_{\mu}$, and $2 \% \nu_{e}+\bar{\nu}_{e}$. In the $0-5 \mathrm{GeV}$ peak energy range, the region which provides greatest sensitivity to $\theta_{34}$ in this Letter, beam interactions are $81 \% \bar{\nu}_{\mu}$. The analysis signal is $\mathrm{NC}$ events, with the sample dominated by $\bar{\nu}$ but with some $\nu$ component that is treated the same; all charged-current (CC) events are considered background.

The detectors are constructed of highly reflective polyvinyl chloride cells [34] with cross-section III.9 cm-by$6.6 \mathrm{~cm}$ and 15.5 (3.8) $\mathrm{m}$ in length for the FD (ND). The cells are formed into 896 (214) planes in the FD (ND), alternating horizontal and vertical relative to the beam axis to enable three-dimensional tracking. Each cell is filled with a mineral-oil based liquid scintillator doped with 5\% pseudocumene [35] and instrumented with a loop of wavelength-shifting fiber, read out at one end using a 32 pixel avalanche photodiode [36]. The FD and ND have masses of $14 \mathrm{kt}$ and $193 \mathrm{t}$, respectively. Custom readout electronics shape and digitize the signal. Pulse height and timing of all energy deposits above a given noise threshold 
are collected in a $550 \mu$ s window encompassing the NuMI beam spill at both detectors. Additionally, data are also collected at the FD using a $10 \mathrm{~Hz}$ minimum bias trigger to sample the cosmogenic background.

The analysis uses data recorded between June 2016 and February 2019, corresponding to an exposure of $12.51 \times$ $10^{20}$ protons-on-target (POT) for the FD. The amount of ND data used corresponds to an exposure of $3.10 \times$ $10^{20}$ POT.

A full simulation of the beam, including all materials and interactions in the beam line, is used to predict the flux at the NOvA detectors. Simulation of the antineutrino production from primary proton-induced hadronic showers and subsequent meson transport and decay is provided by a custom made simulation based on GEANT4 [37,38] with a full geometry of the beam line. The flux is then corrected using a version of the package to predict the flux (PPFX) [39] for the NOvA off-axis beam [40], which modifies the particle production using external hadron production data.

Neutrino interactions in the NOvA detectors are simulated by GENIE [41], with modifications performed by NOvA [42]. The particles are propagated through the detector geometry using GEANT4; the charge deposits are converted to scintillation and transported, along with Cherenkov light, to the front-end electronics using a custom made simulation process. The response of the photodetectors is well understood and used to simulate charge collected by the front-end electronics.

$\mathrm{NC}$ events are characterized by the lack of a charged lepton exiting the vertex and typically consist of diffuse hadronic activity resulting from energy and momentum transfer from the antineutrino to the nucleus. Interactions are identified by requiring a level of activity consistent with these topologies and rejecting $\mathrm{CC}$ events with obvious antimuon tracks or positron showers. The NOvA detectors are well suited to identify $\mathrm{NC}$ interactions since the fine granularity results in the hadronic interaction length of $38 \mathrm{~cm}$ extending to around 10 cells. This enables a good distinction between $\pi^{0} \mathrm{~s}$ and positrons, important in selecting NC events containing hadronic activity and rejecting $\bar{\nu}_{e}$ $\mathrm{CC}$ interactions [43].

The selection was developed on simulated interactions and tested on the ND data, with identical requirements applied to each. The FD has far less overburden than the ND and is exposed to a much higher rate of cosmic-induced interactions; supplementary criteria are, therefore, applied to FD data to remove the cosmogenic backgrounds. The selection applied in this Letter, outlined below, is based on the previous neutrino analysis described in [29].

Hits, cells with activity above threshold, are grouped based on their proximity in space and time to form neutrino events candidates. An event vertex is then reconstructed from these hit collections by projecting back to a common start point. Events are required to have a vertex and be fully contained in the detector. This containment requirement stipulates no activity within an outer volume defined for the ND as $25 \mathrm{~cm}$ from all sides and for the FD as $100 \mathrm{~cm}$ from the top of the detector, $10 \mathrm{~cm}$ from the bottom, and $50 \mathrm{~cm}$ from each of the other sides. Backgrounds arising from neutrino interactions in the material surrounding the ND and resulting in particles, such as neutrons, entering and interacting in the detector are removed by requiring the reconstructed vertex be greater than $100 \mathrm{~cm}$ from the side walls and between 150 and $1000 \mathrm{~cm}$ from the upstream face of the detector.

The primary event classification is provided by a NOvAcustom convolutional neural network (CNN) [44] which identifies interaction topologies from images of the two orthogonal detector views. It is trained separately on simulated FD neutrino- and antineutrino-dominated beam events and cosmic-ray data, and distinguishes the final-state topologies between $\stackrel{(-)}{\nu}_{\mu} \mathrm{CC}, \stackrel{(-)}{\nu}_{e} \mathrm{CC}, \stackrel{(-)}{\nu}_{\tau} \mathrm{CC}, \mathrm{NC}$, and cosmic-induced events. The network learns to extract the characteristic features of the events described above, for example, identifying interactions with charged leptons exiting the vertex as $\mathrm{CC}$ and does not distinguish between interactions from neutrinos and antineutrinos. The separation provided by the CNN for NC events is shown in Fig. 1, and a cut at 0.23 is applied to select events in both detectors. The peak of background events close to one is comprised primarily of high energy deep inelastic scattering interactions, or low energy events with minimal detector activity, neither with an obvious lepton to identify. Interactions occurring in the surrounding rock and subsequently entering the ND are removed by requiring the fractional component of the event momentum which is transverse to the beam direction to be less than $80 \%$. At the $\mathrm{FD}$, similar criteria requiring this to be less than $40 \%$ for showers with a vertex within $2.4 \mathrm{~m}$ from the top of the detector and less than $20 \%$ for a vertex inside $2 \mathrm{~m}$ are used to remove cosmic-induced interactions. For the FD, candidate antineutrino events must not have significant

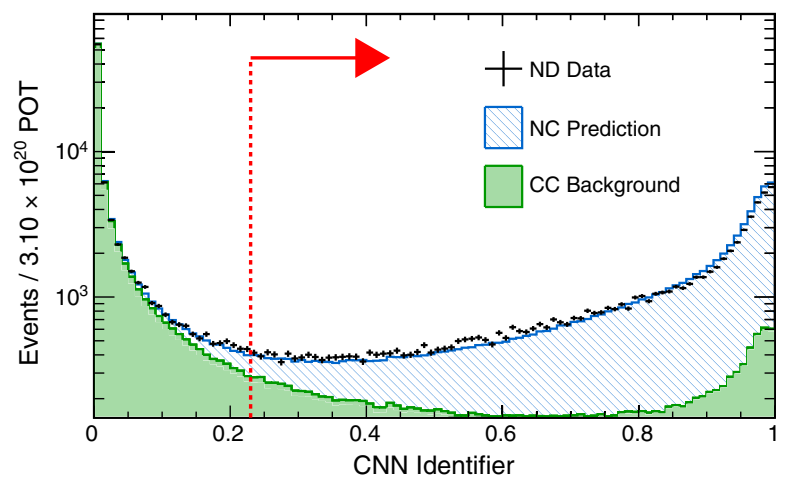

FIG. 1. The CNN likelihood output for neutral-current events used to distinguish between various interaction topologies applied to ND simulation and data. The signal and the dominant predicted background from $\mathrm{CC}$ beam interactions are shown. The red arrow indicates the selected events. 
additional activity within $5 \mathrm{~m}$ and $50 \mathrm{~ns}$ of the final state particles. Events with activity within $200 \mathrm{~cm}$ from the end of the detector must have a sufficient number of downstream planes with hits, compared to the front planes, to ensure showering particles are in the forward direction. A boosted decision tree that considers multiple shower properties is used to further remove cosmic-induced interactions at the FD [45]. Following the application of the full selection, the cosmic background is reduced by over 6 orders of magnitude. The selected sample in the FD simulation is $78 \%$ pure. Two thirds of the remaining backgrounds result from CC interactions where most of the energy is transferred to the hadronic system, and the rest originate from cosmic-induced interactions. The NCcandidate events selected from beam neutrino interactions are $79 \% \bar{\nu}$ and $21 \% \nu$ in the $0-5 \mathrm{GeV}$ peak flux region.

The antineutrino-candidate energy is estimated for NC events by summing the calibrated energy deposited in the interaction and applying a linear scaling from simulation to correct for the unseen outgoing energy. The final reconstructed energy has a resolution of around $30 \%$.

The spectrum of selected NC-candidate events at the ND is shown in Fig. 2. From this, the predicted spectrum at the FD is produced following a data-driven approach in which the ND data are used to constrain the selected simulated events before propagating the resulting distribution to the FD [46]. This procedure uses the simulated ratio between the spectra at the two detectors and takes into account geometric differences and effects of the beam dispersion. The flavor composition and energy of the corrected ND NC-candidate spectrum is inferred from simulation and used to apply oscillations to the events. This approach is effective in partially canceling any correlated uncertainties between the two detectors but results in sensitivity only to active-to-sterile oscillations which develop over larger distances than the ND baseline. In a $3+1$ model, these slower oscillations are driven by mass splittings in the range $0.05 \mathrm{eV}^{2} \leq \Delta m_{41}^{2} \leq 0.5 \mathrm{eV}^{2}$.

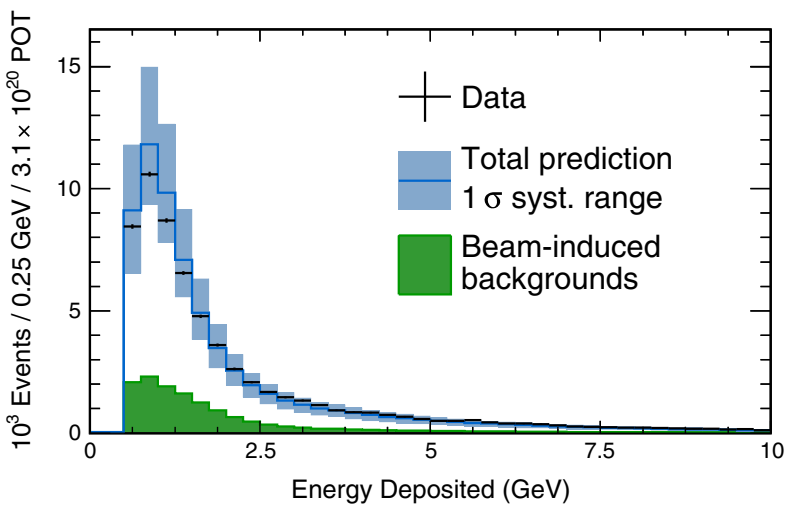

FIG. 2. Spectrum of ND events passing the NC selection. The shaded band shows the total systematic uncertainty. The solid-fill component histogram displays the background contribution from beam $\mathrm{CC}$ interactions.
The dominant uncertainties are summarized in Table I. The largest are attributed to the calibration process which determines the energy scale of the charge collected in each detector cell. An absolute calibration uncertainty of 5\% is used, consistent with the agreement with simulation of proton response in quasielasticlike ND CC interactions. A calibration shape uncertainty to cover charge deposited near the poorly modeled detector edges is evaluated by considering the differences between calibrated and simulated deposits. These uncertainties are applied both as correlated absolute offsets at both detectors and separately as relative differences between them, since they are determined using just the ND. Reducing this large calibration uncertainty is a priority for subsequent analyses, and there are various ongoing efforts, such as improving the analysis procedure and utilizing understanding gained from a test beam program [47].

The simulation contains a modeling of the detector response, including the creation of scintillation and Cherenkov light, its transport to the front-end electronics, and subsequent processing. An uncertainty of $10 \%$ in the normalization of the scintillation light is determined from differences between data and simulation in the number of photoelectrons collected by through-going minimally ionizing particles. The residual uncertainties are quantified using alternate predictions where the scintillation and Cherenkov photon yields in the model are altered within the tolerance of agreement with the ND data while holding the muon response fixed, since that is set by the calibration procedure.

Neutrino interaction model uncertainties are estimated using the GENIE reweighting framework, with additional uncertainties on the modifications of the various interaction models performed by NOvA [42]. An additional uncertainty is considered to account for the differences between the selected spectrum with and without applying these NOvA reweighting modifications. A further $60 \%$ uncertainty on the $\nu_{\tau} \mathrm{CC}$ cross section is allowed, consistent with the most recent measurements [48-51].

TABLE I. The effect of the systematic uncertainties on the NC and $\mathrm{CC}$ expected event rates. The difference is measured as the total change between the shifted event spectrum following the effects of a systematic uncertainty with respect to the nominal spectrum.

\begin{tabular}{lcc}
\hline \hline & NC signal & CC background \\
\cline { 2 - 3 } Uncertainty & Difference $(\%)$ & Difference $(\%)$ \\
\hline Calibration & 13.8 & 9.1 \\
Detector response & 4.9 & 3.8 \\
$\nu$ Interactions & 4.1 & 10.8 \\
Beam & 1.7 & 1.3 \\
Neutron response & 0.5 & 0.2 \\
Tau cross-section & $\ldots$ & 7.6 \\
Total & 15.3 & 16.5 \\
\hline \hline
\end{tabular}


Uncertainties in particle propagation when simulating the beam are provided by the PPFX reweighting framework [39]. Additional transport effects are included by considering the uncertainties in the positioning and modeling of all beam line components including target, focusing horns, and decay volume. The high-energy antineutrinos in the spectrum are produced from kaons, rather than pions, and these hadrons have a $30 \%$ uncertainty on their simulated production. The magnitude of this is motivated by the observed discrepancy with the PPFX prediction in previous MINOS + studies [23].

The fraction of energy carried by neutrons that results in visible signals is uncertain. This is quantified by considering the difference in reconstructed energy between data and simulation for quasielasticlike CC events with one $\mu^{+}$ and a single additional reconstructed object in the final state. This second particle is selected using criteria consistent with having a neutron parent based on energy, displacement from the vertex, and angle. The observed deficit in reconstructed energy for these neutrons is used to estimate the missing energy scale. An uncertainty on the NC-candidate event spectrum is then evaluated using this information to determine the underestimation of the reconstructed antineutrino energy from neutrons.

Each systematic uncertainty is applied to the simulation and used to produce oscillated FD spectra using the datadriven approach previously described. The resulting changes in the event spectrum are taken as the residual energy shifts and included for both signal and background events as additional parameters in the fit. The total uncertainty on simulated FD events following this procedure is shown in Fig. 3.

The event selection and treatment of systematic uncertainties were finalized based on simulation before comparing with the FD data. Upon analyzing the data, 121 NC-candidate events were observed, consistent with expectations from three-flavor oscillations of $122 \pm$ 11 (stat.) \pm 15 (syst.). The composition of events selected

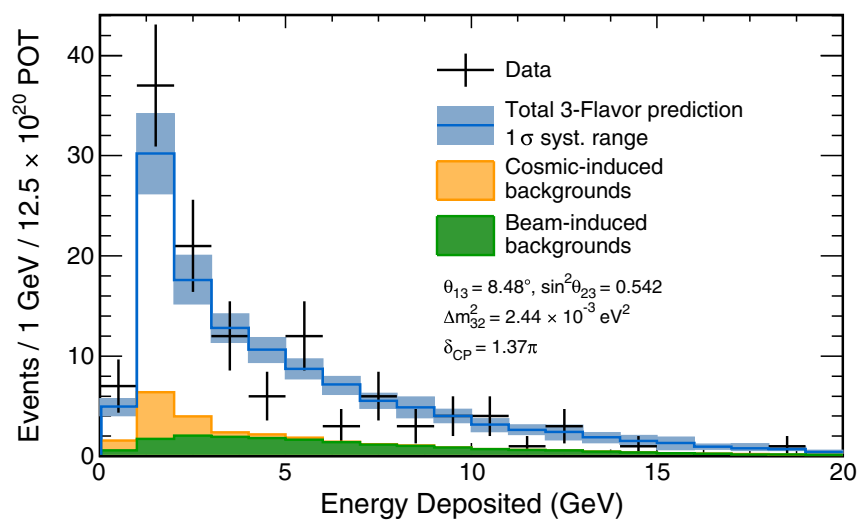

FIG. 3. The reconstructed energy deposited in NC-candidate events in the FD. The observed data spectrum is shown along with the total prediction assuming standard three-flavor oscillations. The contribution from the main backgrounds is also shown. from simulation is detailed in Table II. World-average three-flavor oscillation parameters were used [18], assuming the most conservative case of normal mass ordering and upper $\theta_{23}$ octant. The observed spectrum in Fig. 3 shows good agreement between data and prediction across the range of energies.

To provide a model-independent metric of agreement with an expectation assuming oscillations between only the three active antineutrino species, the ratio $R_{\mathrm{NC}} \equiv[(N-$ $\left.\left.\sum P_{\mathrm{bkg}}\right) / P_{\mathrm{NC}}\right]$ [52] is used to compare the number of data events, $N$, with the predicted number of signal and background events, $P_{\mathrm{NC}}$ and $P_{\mathrm{bkg}}$, respectively. This is equal to unity in the case of no NC disappearance. The obtained value of $0.99 \pm 0.12$ (stat. $)_{-0.16}^{+0.14}$ (syst.) is consistent with no oscillations involving a sterile antineutrino.

A fit considering rate and shape is performed on the spectrum in Fig. 3 to limit the allowed values of the parameters governing sterile oscillations in the $3+1$ framework. The metric

$$
\chi^{2}=\sum_{i} 2\left(P_{i}-N_{i}+N_{i} \ln \frac{N_{i}}{P_{i}}\right)+\sum_{j}\left(\frac{\Delta U_{j}}{\sigma_{j}}\right)^{2}
$$

is minimized, varying the number of events, $P_{i}$, predicted in each FD bin, $i$, and constraining the agreement with data, $N_{i}$, using Gaussian-distributed penalty terms, $U_{j}$, for each systematic uncertainty $j$, each with width $\sigma_{j}$. The mixing angle $\theta_{14}$ and the corresponding phase $\delta_{14}$ are set to zero, consistent with results from solar and reactor experiments [24] and unitarity considerations [53], and the $\delta_{24}$ $C P$-violating phase was included as a nuisance parameter in the fit, allowed to float freely without penalty. The $\delta_{24}$ parameter weakens the limit for large values of both $\theta_{24}$ and $\theta_{34}$ but does not significantly impact the individual limits. The fit is performed at $\Delta m_{41}^{2}=0.5 \mathrm{eV}^{2}$, but the result is valid for the full range $0.05 \mathrm{eV}^{2} \leq$ $\Delta m_{41}^{2} \leq 0.5 \mathrm{eV}^{2}$. Confidence limits are computed using the unified approach of Feldman-Cousins [54] and are shown in Fig. 4.

Profiling over each sterile mixing angle, in turn, provides limits of $\theta_{24}<25^{\circ}$ and $\theta_{34}<32^{\circ}$ at $90 \%$ C.L. Table III shows these constraints, together with the inferred matrix element values, alongside results from other experiments.

TABLE II. Predicted number of events selected as NC candidates at the far detector using the data-driven procedure. The systematic uncertainty on each of the components is shown.

\begin{tabular}{lc}
\hline \hline NC signal & $95.5 \pm 14.6$ \\
$\nu_{\mu}$ CC background & $12.2 \pm 2.0$ \\
$\nu_{e}$ CC background & $3.6 \pm 0.6$ \\
$\nu_{\tau}$ CC background & $2.2 \pm 0.4$ \\
Cosmic background & $8.7 \pm 0.4$ \\
Total & $122.2 \pm 14.8$ \\
\hline \hline
\end{tabular}



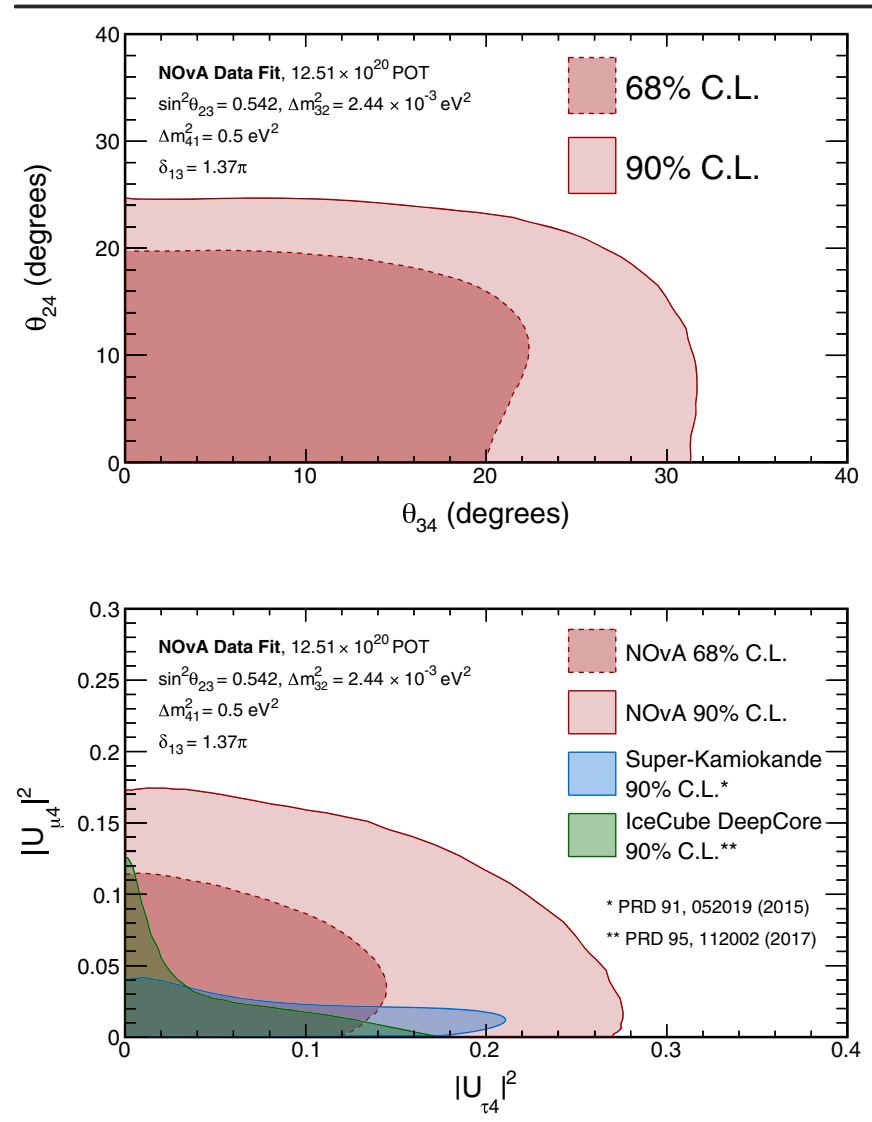

FIG. 4. Allowed regions in parameter space defined by the mixing elements describing neutrino oscillations in a $3+1$ model. Top: The $68 \%$ and $90 \%$ allowed values of the mixing angles $\theta_{24}$ and $\theta_{34}$. Bottom: The matrix element space, with limits from comparable measurements from Super-Kamiokande [25] and IceCube DeepCore [27]. The limits from Super-Kamiokande and IceCube are set using atmospheric neutrinos, a sample which is majority neutrinos but with a non-negligible antineutrino component. The NOvA limits are the first set from studying an antineutrino-dominated sample.

TABLE III. Upper 90\% C.L. limits on the mixing angles $\theta_{24}$ and $\theta_{34}$, with the associated values of the matrix elements, for this result (NOvA $\bar{\nu}$ ) and comparable measurements from NOvA $\nu$ [29], MINOS+ [23], T2K [28], Super-Kamiokande [25], IceCube [26], and IceCube-DeepCore [27]. The limits are shown for $\Delta m_{41}^{2}=0.5 \mathrm{eV}^{2}$ for all experiments, except IceCube-DeepCore and $\mathrm{T} 2 \mathrm{~K}$, where the results are reported for $\Delta m_{41}^{2}=1.0 \mathrm{eV}^{2}$.

\begin{tabular}{lrlll}
\hline \hline & $\theta_{24}$ & $\theta_{34}$ & $\left|U_{\mu 4}\right|^{2}$ & $\left|U_{\tau 4}\right|^{2}$ \\
\hline NOvA $(\bar{\nu})$ & $25^{\circ}$ & $32^{\circ}$ & 0.175 & 0.276 \\
NOvA $(\nu)$ & $20.8^{\circ}$ & $31.2^{\circ}$ & 0.126 & 0.268 \\
MINOS/MINOS + & $4.4^{\circ}$ & $23.6^{\circ}$ & 0.006 & 0.160 \\
T2K & $18.4^{\circ}$ & $45.0^{\circ}$ & 0.1 & 0.5 \\
Super-Kamiokande & $11.7^{\circ}$ & $25.1^{\circ}$ & 0.041 & 0.180 \\
IceCube & $4.1^{\circ}$ & $\ldots$ & 0.005 & $\ldots$ \\
IceCube-DeepCore & $19.4^{\circ}$ & $22.8^{\circ}$ & 0.11 & 0.150 \\
\hline \hline
\end{tabular}

This result is compatible with world limits from analyses reporting no evidence of sterile neutrino oscillations, shown in Fig. 4, and is the first from an analysis of antineutrinos over a long baseline.

To conclude, 121 neutral-current candidates are observed at the far detector compared with $122 \pm 11$ (stat.) \pm 15 (syst.) events predicted assuming mixing only occurs between active species. This is the first accelerator-based search for oscillations into sterile neutrinos over long baselines using an antineutrino-dominated beam, and no evidence of a depletion of the active flavors is observed. The measurement reported in this Letter enhances the understanding of antineutrino oscillations in the context of sterile neutrino anomalies. Looking forward, NOvA plans to increase its antineutrino dataset by a factor of around 2.5, complementing a comparable neutrino sample, which will facilitate improved sterile neutrino searches and enable more precise measurements of the governing parameters.

This document was prepared by the NOvA Collaboration using the resources of the Fermi National Accelerator Laboratory (Fermilab), a U.S. Department of Energy, Office of Science, HEP User Facility. Fermilab is managed by Fermi Research Alliance, LLC (FRA), acting under Contract No. DE-AC02-07CH11359. This work was supported by the U.S. Department of Energy; the U.S. National Science Foundation; the Department of Science and Technology, India; the European Research Council; the MSMT CR, GA UK, Czech Republic; the RAS, MSHE, and RFBR, Russia; CNPq and FAPEG, Brazil; UKRI, STFC and the Royal Society, United Kingdom; and the state and University of Minnesota. We are grateful for the contributions of the staffs of the University of Minnesota at the Ash River Laboratory, and of Fermilab.

* Corresponding author. michael.wallbank@uc.edu

[1] Y. Fukuda et al. (Super-Kamiokande Collaboration), Phys. Rev. Lett. 81, 1562 (1998).

[2] Q. R. Ahmad et al. (SNO Collaboration), Phys. Rev. Lett. 89, 011301 (2002).

[3] T. Araki et al. (KamLAND Collaboration), Phys. Rev. Lett. 94, 081801 (2005).

[4] M. H. Ahn et al. (K2K Collaboration), Phys. Rev. D 74, 072003 (2006).

[5] D. G. Michael et al. (MINOS Collaboration), Phys. Rev. Lett. 97, 191801 (2006).

[6] K. Abe et al. (T2K Collaboration), Phys. Rev. Lett. 107, 041801 (2011).

[7] F. P. An et al. (Daya Bay Collaboration), Phys. Rev. Lett. 108, 171803 (2012).

[8] J. K. Ahn et al. (RENO Collaboration), Phys. Rev. Lett. 108, 191802 (2012).

[9] Y. Abe et al. (Double Chooz Collaboration), J. High Energy Phys. 10 (2014) 086. 
[10] N. Agafonova et al. (OPERA Collaboration), Phys. Rev. Lett. 115, 121802 (2015).

[11] P. Adamson et al. (NOvA Collaboration), Phys. Rev. Lett. 116, 151806 (2016).

[12] A. Aguilar et al. (LSND Collaboration), Phys. Rev. D 64, 112007 (2001).

[13] A. Aguilar-Arevalo et al. (MiniBooNE Collaboration), Phys. Rev. Lett. 121, 221801 (2018).

[14] P. Huber, Phys. Rev. C 84, 024617 (2011).

[15] T. A. Mueller, D. Lhuillier, M. Fallot, A. Letourneau, S. Cormon, M. Fechner, L. Giot, T. Laserre, J. Martino, G. Mention et al., Phys. Rev. C 83, 054615 (2011).

[16] M. A. Acero, C. Giunti, and M. Laveder, Phys. Rev. D 78, 073009 (2008).

[17] C. Giunti and M. Laveder, Phys. Rev. C 83, 065504 (2011).

[18] M. Tanabashi et al. (Particle Data Group), Phys. Rev. D 98, 030001 (2018).

[19] K. Abazajian et al., arXiv:1204.5379.

[20] B. Pontecorvo, Zh. Eksp. Teor. Fiz. 34, 247 (1957) [Sov. Phys. JETP 7, 172 (1958)].

[21] Z. Maki, M. Nakagawa, and S. Sakata, Prog. Theor. Phys. 28, 870 (1962).

[22] H. Harari and M. Leurer, Phys. Lett. B 181, 123 (1986).

[23] P. Adamson et al. (MINOS+ Collaboration), Phys. Rev. Lett. 122, 091803 (2019).

[24] F. P. An et al. (Daya Bay Collaboration), Phys. Rev. Lett. 115, 111802 (2015).

[25] K. Abe et al. (Super-Kamiokande Collaboration), Phys. Rev. D 91, 052019 (2015).

[26] M. G. Aartsen et al. (IceCube Collaboration), Phys. Rev. Lett. 117, 071801 (2016).

[27] M. G. Aartsen et al. (IceCube Collaboration), Phys. Rev. D 95, 112002 (2017).

[28] K. Abe et al. (T2K Collaboration), Phys. Rev. D 99, 071103 (R) (2019).

[29] P. Adamson et al. (NOvA Collaboration), Phys. Rev. D 96, 072006 (2017).

[30] A. A. Aguilar-Arevalo et al. (MiniBooNE Collaboration), Phys. Rev. Lett. 105, 181801 (2010).

[31] A. Aguilar-Arevalo et al. (MiniBooNE Collaboration), Phys. Rev. Lett. 110, 161801 (2013).

[32] P. Adamson et al. (MINOS Collaboration), Phys. Rev. Lett. 117, 151803 (2016).

[33] P. Adamson et al. (MINOS Collaboration), Nucl. Instrum. Methods Phys. Res., Sect. A 806, 279 (2016).
[34] R. L. Talaga, J. J. Grudzinski, S. Phan-Budd, A. Pla-Dalmau, J. E. Fagan, C. Grozis, and K. M. Kephart, Nucl. Instrum. Methods Phys. Res., Sect. A 861, 77 (2017).

[35] S. Mufson et al., Nucl. Instrum. Methods Phys. Res., Sect. A 799, 1 (2015).

[36] The NOvA APD is a custom variant of the Hamamatsu S8550, https://web.archive.org/web/20210201152847/https:// www.hamamatsu.com/us/en/product/type/S8550-02/index .html.

[37] S. Agostinelli et al., Nucl. Instrum. Methods Phys. Res., Sect. A 506, 250 (2003).

[38] J. Allison et al., IEEE Trans. Nucl. Sci. 53, 270 (2006).

[39] L. Aliaga Soplin, Ph.D. thesis, William-Mary College, 2016 [Report No. FERMILAB-THESIS-2016-03].

[40] NuMI Neutrino Flux for 2017 NOvA Analyses, https:// novaexperiment.fnal.gov/data-releases/.

[41] C. Andreopoulos et al., Nucl. Instrum. Methods Phys. Res., Sect. A 614, 87 (2010).

[42] M. Acero et al. (NOvA Collaboration), Eur. Phys. J. C 80, 1119 (2020).

[43] NOvA Technical Design Report, Report No. FERMILABDESIGN-2007-01, https://www.osti.gov/biblio/935497, 2007.

[44] A. Aurisano, A. Radovic, D. Rocco, A. Himmel, M. D. Messier, E. Niner, G. Pawloski, F. Psihas, A. Sousa, and P. Vahle, J. Instrum. 11, P09001 (2016).

[45] S. Yang, Ph.D. thesis, Cincinnati University, 2019 [Report No. FERMILAB-THESIS-2019-09].

[46] M. A. Acero et al., Phys. Rev. D 98, 032012 (2018).

[47] M. Wallbank (NOvA Collaboration), Proc. Sci., ICHEP2020 (2020) 188.

[48] K. Kodama et al. (DONuT Collaboration), Phys. Rev. D 78, 052002 (2008).

[49] Z. Li et al. (Super-Kamiokande Collaboration), Phys. Rev. D 98, 052006 (2018).

[50] N. Agafonova et al. (OPERA Collaboration), Phys. Rev. Lett. 120, 211801 (2018).

[51] M. G. Aartsen et al. (IceCube Collaboration), Phys. Rev. D 99, 032007 (2019).

[52] P. Adamson et al. (MINOS Collaboration), Phys. Rev. Lett. 101, 221804 (2008).

[53] S. Parke and M. Ross-Lonergan, Phys. Rev. D 93, 113009 (2016).

[54] G. J. Feldman and R. D. Cousins, Phys. Rev. D 57, 3873 (1998). 